\title{
Fundamental Properties of Belt Nip Twister for Yarn Texturing
}

\author{
By Tooru Takahashi and Teiryo Kojima, Members, TMSJ
}

\author{
Fibers and Textiles Research Laboratories, Toray Industries Inc., Sonoyama 3-3-7, Otsu-shi, 520 Japan
}

Based on the Journal of the Textile Machinery Society of Japan, Transactions, Vol. 37, No. 12, P487-P491 (1984-12)

\begin{abstract}
Two parameters, twist efficiency and yarn delivery speed ratio, were adopted to clear the properties of the twisting and the yarn delivery of a blet nip twister to get a satisfactory twisting condition.

Although these parameters varied with twister condition $(B / Y$ ratio and the intersecting angle between the belts), the relationship between the detwisting tension/twisting tension ratio and the yarn delivery speed ratio is approximately on a master curve. So, when the yarn delivery speed ratio is equal to one, the $B / Y$ ratio is given by: $1 /(1+\varepsilon) \cdot 1 / \cos (\theta / 2)$

where $\varepsilon$ : elongation ratio of untwisting, $\theta$ : intersecting angle between the belts
\end{abstract}

\section{Introduction}

Nowadays POY-DTY and friction twisting have become more popular, and the tape-up speed of false twist texturing has reached $900-1,200 \mathrm{~m} / \mathrm{min}$ in catalogue data and $600-800 \mathrm{~m} / \mathrm{min}$ in real production.

In this background, the nip-type yarn twisting mechanism, that was unveiled by Murata Machinery, Ltd. in 1979 and by Barmag Maschinenfabrik, A.G. in 1982, got a great interest in this business world.

In this paper, some experimental results of the belt nip twister concerning with the relationship between the twister conditions and the twister properties are reported.

\section{Test Machine}

Mach Crimper 333-Il with 24 texturing positions by Murata Machinery, Ltd. was used in the original specifications.

\section{Purpose and Test Method}

Yarn twisting property and yarn delivery property should be influenced with yarn radial portion $V_{T}$ and yarn longitudinal portion $V_{Y}$ of twister speed. Here, symbols are defined as shown in Fig. 1:

$$
\begin{aligned}
V_{T} & =V \sin \frac{\theta}{2} \\
V_{Y} & =V \cos \frac{\theta}{2}
\end{aligned}
$$

Then, we tried to analyze the properties of the belt nip twister by measuring twist density, twisting tension and detwisting tension under the various conditions of the belt speed $V$ and the intersecting angle of the belts $\theta$. Then the velocity ratio $(V / v)$ of belt speed to yarn speed is adopted in stead of the belt speed $V$, and it is called $B / Y$ ratio, Polyester POY as feeder yarns are:

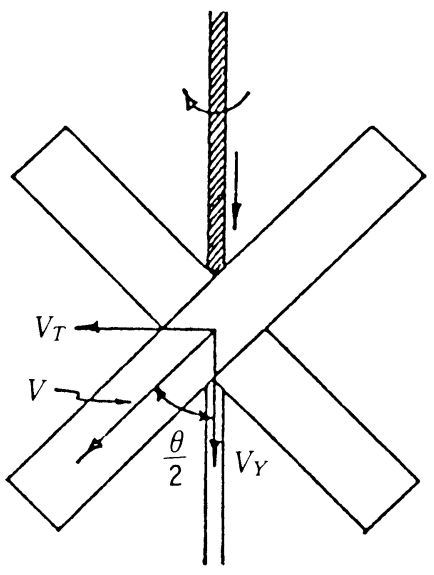

Fig. 1 Vector analysis of speeds of the twister and the yarn

- 75 denier (after draw texturing), 24 filament semidull and round cross section,

- 150 denier (after draw texturing), 48 filaments semidull and round cross section.

\section{Test Result and Consideration}

\subsection{Twisting properties}

The relations between the false twisting number and the intersecting angle between the belts and the $B / Y$ ratio are shown in Fig. 2. The false twisting number increases with increase of $\theta$ and $B / Y$ ratio. These results were easily expected from eq. (1).

\subsection{Twisting efficiency}

In an ideal condition of twisting there will be completely no slip between the yarn and the twister along the yarn twisting direction and the yarn advancing direction. Then we try to estimate the amount of slip between the yarn and the twister in 


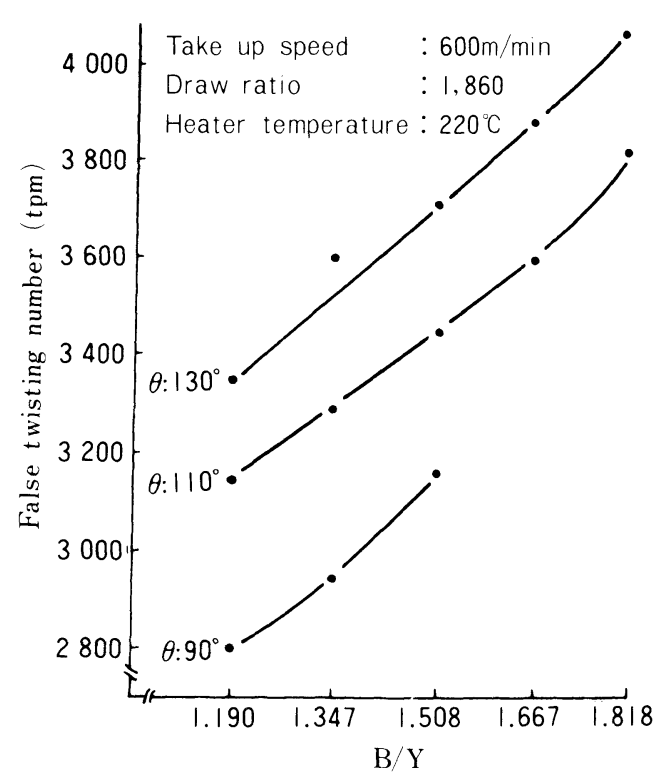

(a) Feeder yarn :75D $\begin{array}{ll}\text { Take up speed } & : 600 \mathrm{~m} / \mathrm{min} \\ \text { Draw ratio } & : 1,860 \\ \text { Heater temperature }: 220^{\circ} \mathrm{C}\end{array}$

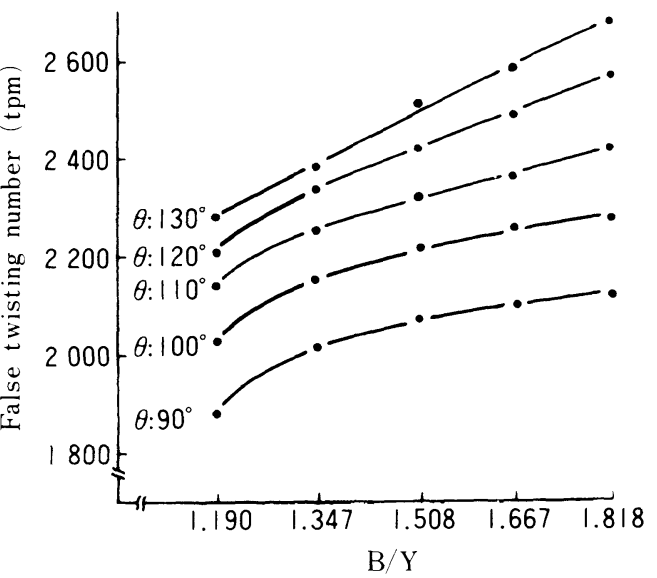

(b) Feeder yarn: 150D

Fig. 2 Relations between the false twisting number and the intersecting angle between the belts $\theta$ and $B / Y$ ratio

the belt nip twister. When yarn model around the twister is shown in Fig. 3, and symbols are defined as follows:

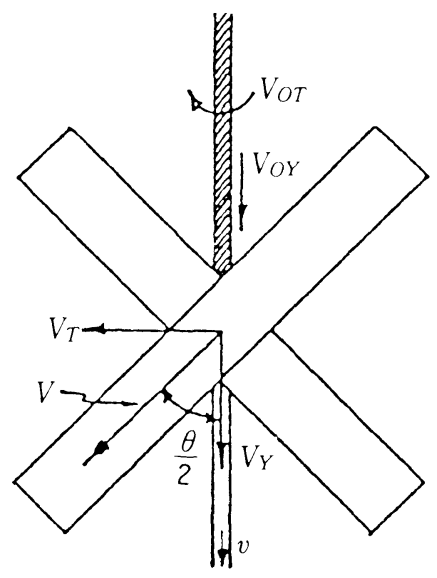

Fig. 3

$v:$ take-up speed

$d$ : diameter of the twisted yarn

$T$ : twist number per unit length of the twisted yarn

$D y:$ denier of textured (detwisted) yarn

$\varepsilon$ : elongation ratio at detwisting,

the advancing velocity of the twisted yarn $V_{O Y}$ at the twister is

$$
V_{O Y}=v / 1+\varepsilon
$$

The rotating velocity of the twisted yarn $V_{O T}$ at the twister is obtained from the next equation:

$$
V_{0 T}=\pi \cdot d \cdot T \cdot V_{0 Y}=\pi \cdot d \cdot T \cdot v / 1+\varepsilon \quad \cdot \cdot(4)
$$

When the twisting efficiency is defined as $V_{O T} / V_{T}$, we have from eqs. (1) and (4)

$$
V_{0 T} / V_{T}=\pi \cdot d \cdot T / 1+\varepsilon \cdot v / V \cdot 1 / \sin \frac{\theta}{2} \cdots \cdots(5)
$$

Therefore, we can calculate the twisting efficiency from eq. (5) by measuring $d, T$ and $\varepsilon$. Assuming that there is no space among filaments of a twisted yarn in a shape of a right cylinder with density 1.38 , the radius of the twisted yarn is calculated as follows:

$$
\varepsilon=0.02 \sqrt{\frac{D_{y}(1+\varepsilon)}{9 \times 10^{5} \times \pi \times 1.38}}
$$

The twist number of the twisted yarn $T$ is decided by measuring the twist density of the twisted yarn under tension $0.1 \mathrm{~g} / \mathrm{d}$, and expressed in the unit of turns $/ \mathrm{m}$. The elongation ratio at detwisting $\varepsilon$ is expressed by $\varepsilon=\frac{l_{2}-l_{1}}{l_{1}}$

where $l_{1}$ and $l_{2}$ are the yarn lengths after twisted and after detwisted, respectively.

The calculated twist efficiencies from eq. (5) and the data in Fig. 2 are shown in Fig. 4.

In cases of 75 denier and 150 denier, the twist efficiencies are scarcely influenced with the intersecting angles between the belts, and drop with increase of $B / Y$ ratio. Twist efficiencies of 150 denier are lower than those of 75 denier.

\subsection{Yarn advancing property}

Figure 5 shows the twisting and the detwisting tensions of the yarn in relation with the $B / Y$ ratio. The tension ratio of detwisting/twisting is shown in Fig. 6. These data were obtained from the same experiment shown in Fig 2.

As predicted from equation (2), when the $B / Y$ ratio increases or the intersecting angle between the belts decreases, the yarn longitudinal portion $V_{Y}$ of the twister speed and the twisting tension increase, and the detwisting tension decreases. Thus, the tension ratio of detwisting/twisting decreases.

\subsection{Yarn advancing speed ratio}

We try to estimate the amount of slip, in the direction of yarn advancing, between the yarn and the twister as well as the twisting efficiency.

From eqs. (2) and (3), $V_{O Y} / V_{Y}$ can be calculated and defined as the yarn advancing speed ratio as follows:

$$
V_{O Y} / V_{Y}=v / V \cdot 1 / 1+\varepsilon \cdot 1 / \cos \frac{\theta}{2}
$$

Figure 7 shows the results of the calculation of the yarn advancing speed ratio by combining eq. (7) with the elongation ratio at detwisting $\varepsilon$ got when the twist number is observed in Fig. 2. In the cases of 75 and 150 denier, the yarn advancing 


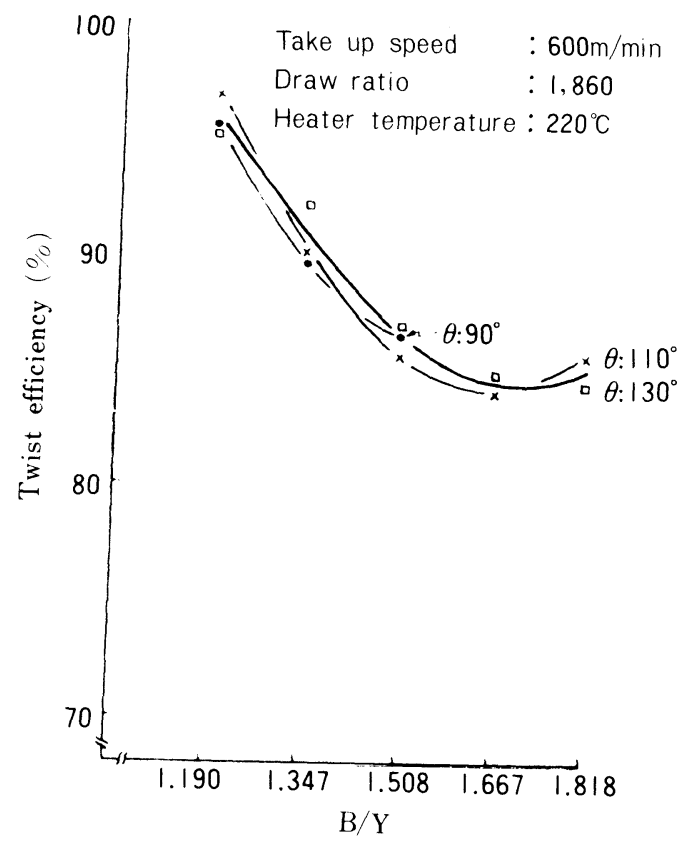

(a) Feeder yarn : 75D

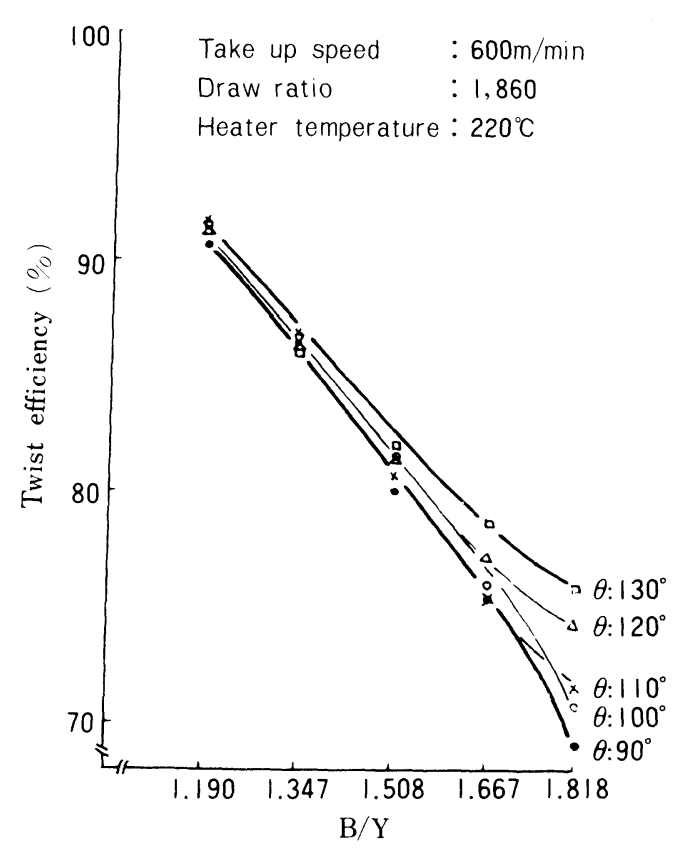

(b) Feeder yarn: 150D

Fig. $4 \theta, B / Y$ ratio and twist efficiencies

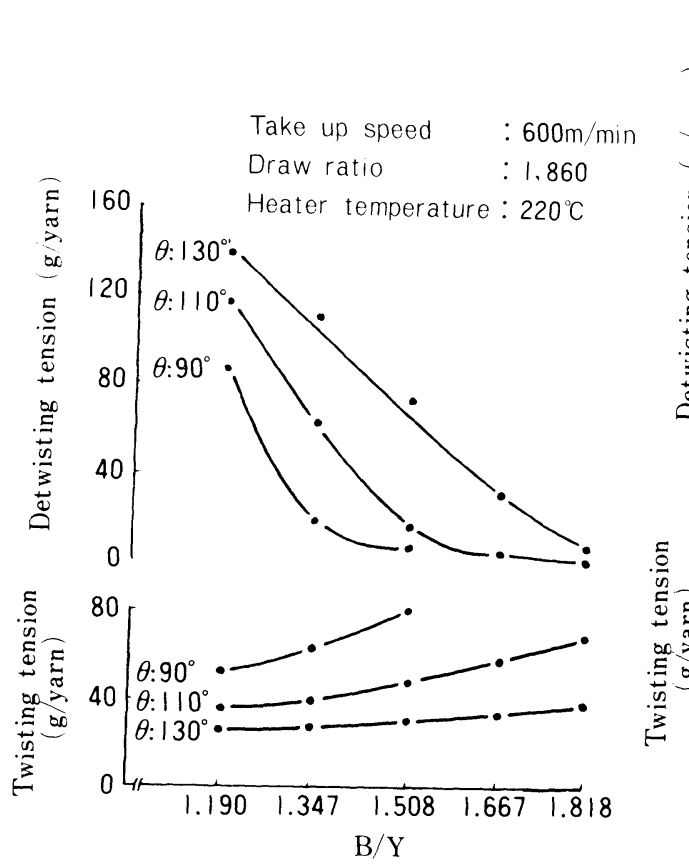

(a) Feeder yarn : 75D
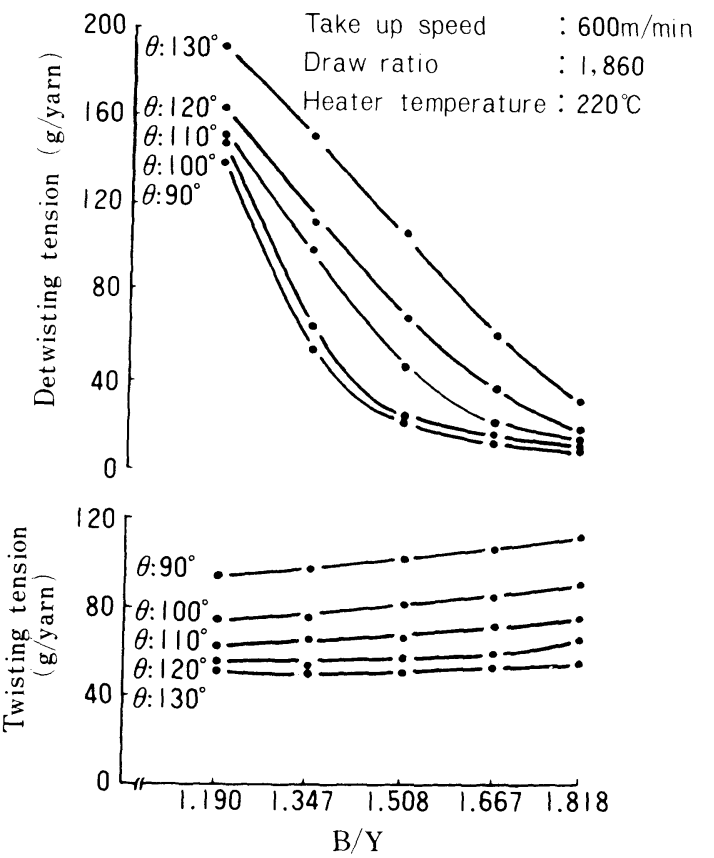

(b) Feeder yarn: 150D

Fig. $5 \theta, B / Y$ and twisting tension and detwisting tension

speed ratio decrease with increase of the $B / Y$ ratio or with decrease of the intersecting angle between the belts $\theta$. The ideal yarn advancing condition may be when the yarn advancing speed ratio is equal to one. So, we put $V_{O Y} / V_{Y}=1$ in eq. (7), and it goes:

$$
\begin{aligned}
& v / V \cdot 1 / 1+\varepsilon \cdot 1 / \cos \frac{\theta}{2}=1 \\
& V / v=1 / 1+\varepsilon \cdot 1 / \cos \frac{\theta}{2} \ldots .
\end{aligned}
$$

Figure 8 shows the relationship between the intersecting angle between the belts $\theta$ and the $B / Y$ ratio when the yarn advancing speed ratio is one in Fig. 7.

In the cases of 75 and 150 denier, the $B / Y$ ratio increases with $\theta$ when the yarn advancing speed ratio is 1 . In Fig. 8, curves (a) and (b) almost coincide. So, the relationship between $\theta$ and the $B / Y$ ratio where the yarn advancing speed ratio is one seems to be scarcely influenced with yarn denier. Also, Fig. 8 suggests the 


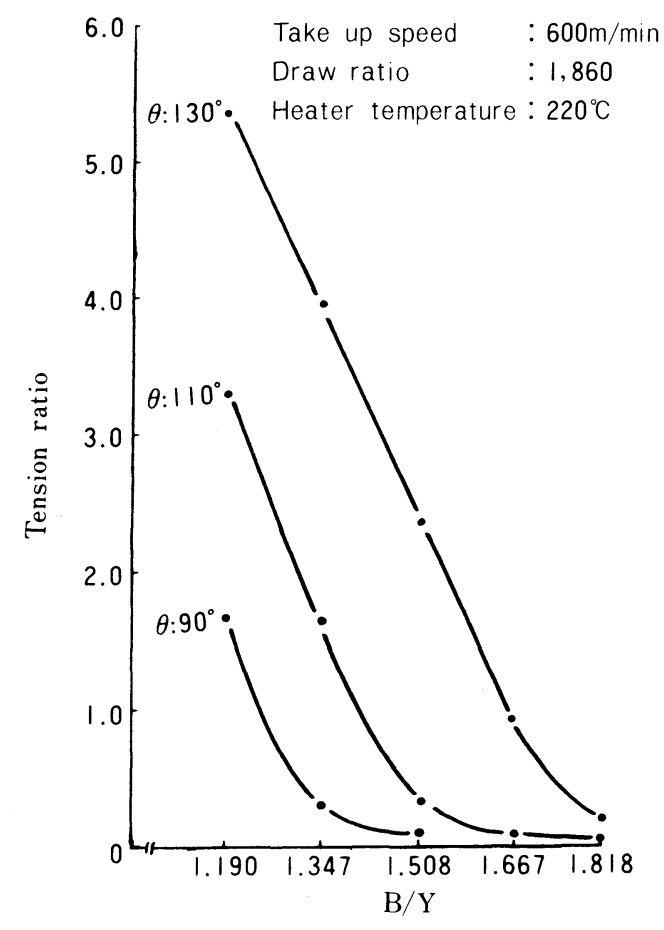

(a) Feeder yarn :75D $\begin{array}{ll}\text { Take up speed } & : 600 \mathrm{~m} / \mathrm{min} \\ \text { Draw ratio } & : 1,860\end{array}$

Heater temperature : $220^{\circ} \mathrm{C}$

Fig. $6 \theta, B / Y$ and tension ratio of detwisting/twisting

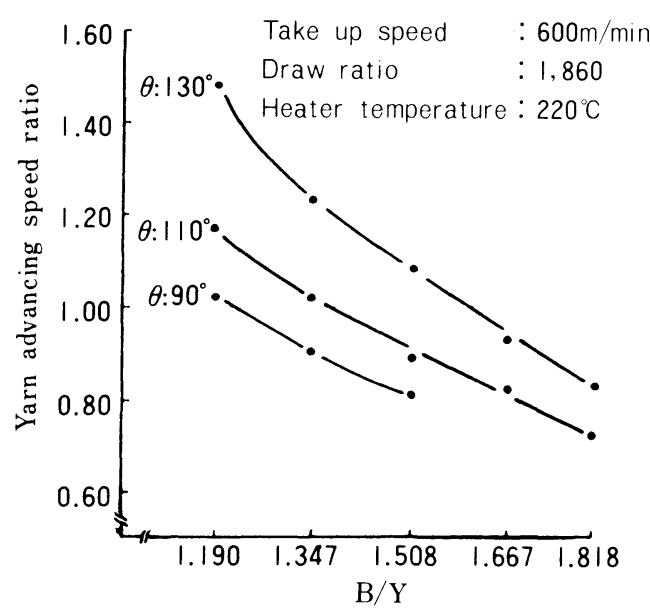

(a) Feeder yarn :75D

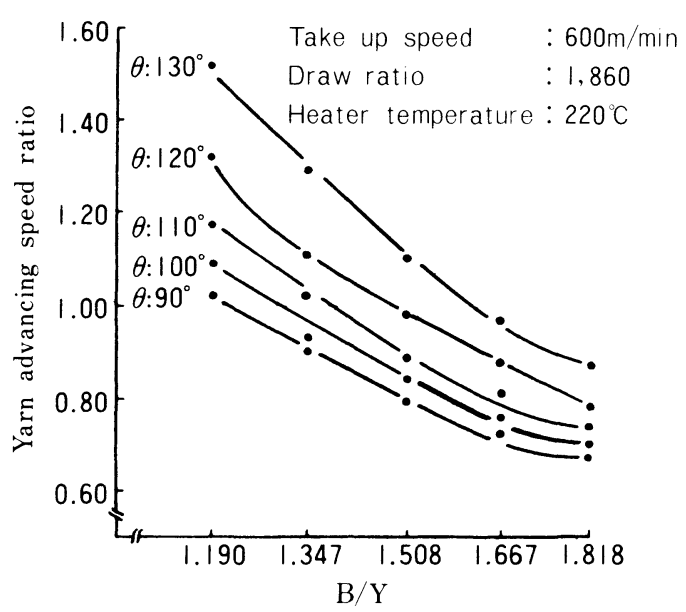

(b) Feeder yarn: 150D

Fig. $7 \theta, B / Y$ and yarn advancing speed ratio

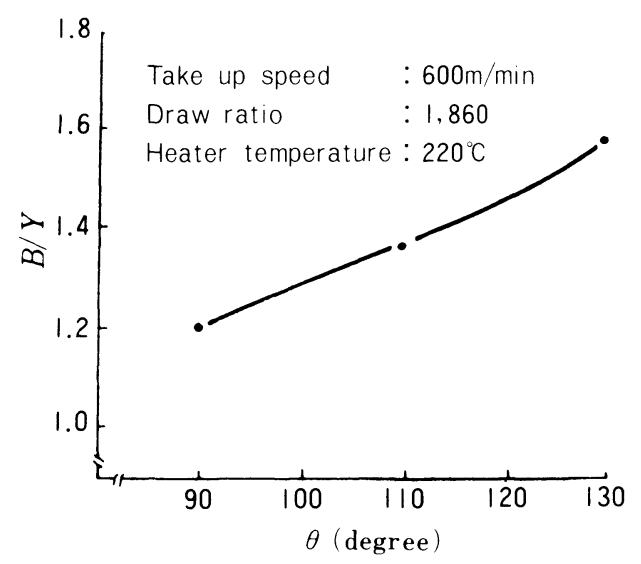

(a) Feeder yarn: 75D

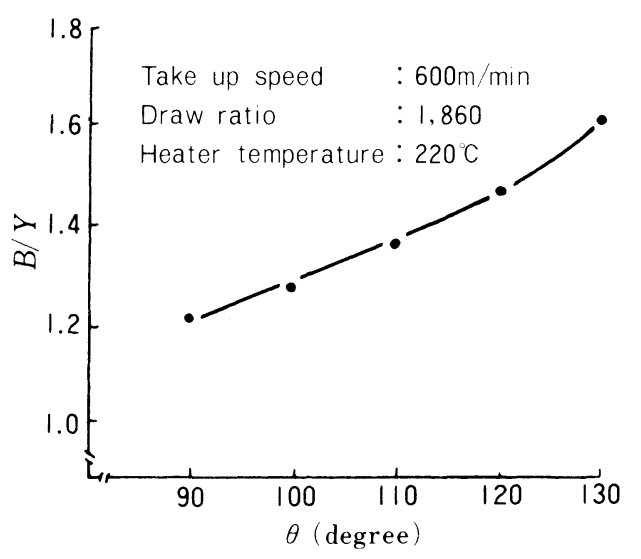

(b) Feeder yarn: 150D

Fig. $8 \theta$ and $B / Y$ when the yarn advancing speed ratio is one 


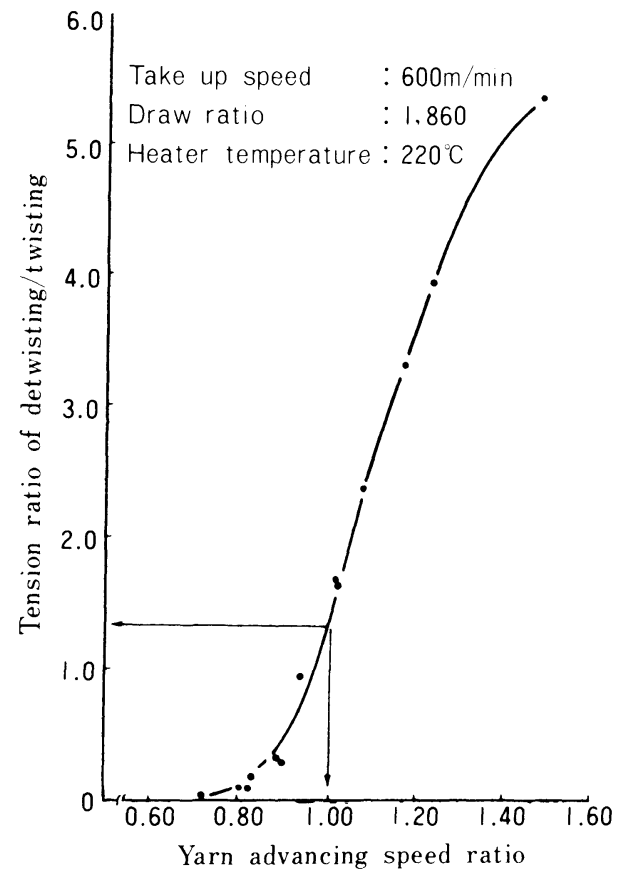

(a) Feeder yarn : 75D
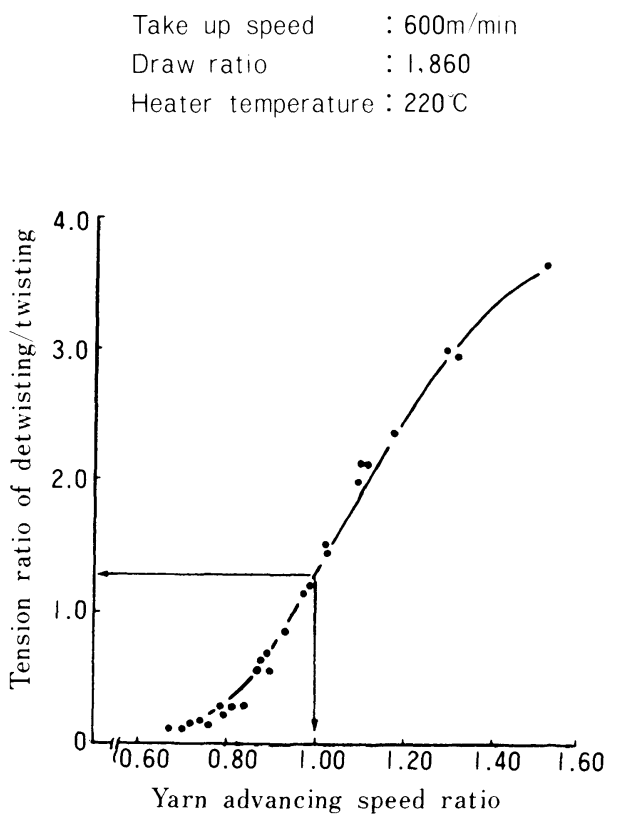

(b) Feeder yarn : 150D

Fig. 9 Yarn advancing speed ratio and tension ratio of detwisting/twisting

existance of the optimum relationship between $\theta$ and the $B / Y$ ratio in order to get a normal false twist textured yarn.

\subsection{Yarn advancing speed ratio and tension ratio of de- twisting/twisting}

From the yarn advancing speed ratio shown in Fig. 7 and the tension ratio detwisting/twisting shown in Fig. 6, the relationship among them shown in Fig. 9 is obtained. Every points in Fig. 9 lines on a master curve having no connection with $\theta$ or the $B / Y$ ratio.

The shape of this curve seems to be influenced with the streched stress of the twisted yarn and the crimping force of the untwisted yarn. In the cases of 75 denier and 150 denier, the tension ratio of detwisting/twisting is equal to 1.2-1.3 when the yarn advancing speed ratio is one in Fig. 9(a) and (b).

\section{Conclusion}

(1) The false twist number increases with increase of the $B / Y$ ratio or the interesting angle between belts $\theta$. The twisting efficiency is scarcely influenced with $\theta$, but decreases with increase of the $B / Y$ ratio.

(2) In the usual condition, the twisting efficiency is $85-90 \%$.

(3) The tension ratio of detwisting/twisting decreases with increase of the $B / Y$ ratio and with decrease of the intersecting angle between the belts $\theta$.

(4) The relationship between the yarn advancing speed ratio and the tension ratio of detwisting/twisting draws a master curve.

(5) Combinations of the $B / Y$ ratio and $\theta$ when the yarn advancing speed ratio is unit can be calculated with

$$
V / v=1 / 1+\varepsilon \cdot 1 / \cos \frac{\theta}{2}
$$

or can be experimentally obtained from the point where the tension ratio of detwisting/twisting is $1.2-1.3$ in Fig. 9. (Addition)

This paper is an added and amended one of a content presented in the 36th annual conference of this institute at June in 1983. 